\title{
Circular RNA: biogenesis, degradation, functions and potential roles in mediating resistance to anticarcinsuogens
}

\author{
Xiuchao Geng $g^{\ddagger, 1}$, Youchao Jia ${ }^{\ddagger 2}$, Yuhao Zhang ${ }^{3}$, Liang Shi ${ }^{4}$, Qiang $\mathrm{Li}^{5}$, Aimin Zang ${ }^{\star *, 2}$ \& \\ Hong Wang*,1 \\ ${ }^{1}$ Faculty of Integrated Traditional Chinese \& Western Medicine, Hebei University of Chinese Medicine, Shijiazhuang 050091, PR \\ China \\ ${ }^{2}$ Department of Medical Oncology, Hebei Key Laboratory of Cancer Radiotherapy \& Chemotherapy, Affiliated Hospital of Hebei \\ University, Baoding 071000, PR China \\ ${ }^{3}$ Department of Neurosurgery, Affiliated Hospital of Hebei University, Baoding 071000, PR China \\ ${ }^{4}$ Endoscopy Division, Department of General Surgery, Cangzhou Central Hospital, Cangzhou 061000, PR China \\ ${ }^{5}$ Faculty of Acupuncture-Moxibustion \& Tuina, Hebei University of Chinese Medicine, Shijiazhuang 050091, PR China \\ *Author for correspondence: bossw@vip.sina.com \\ **Author for correspondence: booszam@sina.com \\ $\ddagger$ Authors contributed equally.
}

Aim: This review aims to systematically describe the biogenesis and degradation of circular RNAs (circRNAs), discusses the major functions of circRNAs, introduces the mechanisms by which circRNAs play a role in cancer, comprehensively summarize the relationship between circRNAs and anticarcinogen resistance as well as underlying specific mechanisms in multiple cancers. Materials \& methods: We screened and analyzed large quantity of scientific papers which associated with circRNAs, noncoding RNAs, function, cancer, drug resistance and chemoresistance, and then summarized in Figures 1 \& 2 \& Table 1. Results \& conclusion: The biogenesis, degradation and function of circRNAs are specially compared with other noncoding RNAs, it can affect cancer pathogenesis and progression and are implicated in mediating resistance to various anticarcinogens in various types of cancer.

First draft submitted: 6 October 2019; Accepted for publication: 19 November 2019; Published online: 6 December 2019

Keywords: anticarcinogens $\bullet$ biogenesis $\bullet$ cancer $\bullet$ circRNA $\bullet$ circular RNA $\bullet$ degradation $\bullet$ drug resistance $\bullet$ function - mechanism • noncoding RNA

Noncoding RNAs (ncRNAs) account for 95\% of the total RNA in the eukaryotic genome. Although circular RNA (circRNA), a covalently closed ncRNA, was initially considered a splicing error, its important role in gene regulation is increasingly recognized. CircRNAs are generally generated via back-splicing or exon skipping of precursor mRNAs (pre-mRNAs), a process that differs from canonical splicing. Interestingly, circRNAs are abundant in the cytoplasm, evolutionarily conserved among species and relatively stable compared with their linear counterparts. These features endow circRNAs with many potential functions, such as regulating the expression of parental genes, regulating alternative splicing or translation, acting as miRNA sponges (competing endogenous RNA [ceRNA] networks) or RNA-binding protein (RBP) sponges, being translated into peptides/proteins (only a few circRNAs) and producing some pseudogenes.

NcRNAs are closely linked to most common diseases, especially human cancers, and are involved in mediating drug resistance in many kinds of cancer. Currently, the majority of reported studies are on miRNAs [1] and long noncoding RNAs (lncRNAs) [2]. However, in addition to miRNA and lncRNA, another type of ncRNA, circRNA, has recently become a research focus. CircRNAs can participate in a series of physiological and pathological processes. An increasing number of studies have revealed that circRNAs have different expression levels in multiple cancers and are closely related to the occurrence and progression (development, invasion and metastasis) of cancer. Interestingly, only a few studies have reported that circRNAs have crucial functions in mediating resistance to anticarcinogens in cancer as identified by advanced experimental techniques, including high-throughput RNA sequencing, CRISPR 
screening, and genome-wide association studies, and these findings offer new diagnostic markers and therapeutic targets in cancer. Currently, the contribution of circRNAs to the development of anticarcinogen resistance in the context of tumors has not been fully described, and the mechanism of circRNAs in anticarcinogen resistance has not been elucidated. This review systematically describes the mechanism of circRNAs biogenesis and degradation, discusses the major functions of circRNAs, introduces the mechanisms by which circRNAs play a role in cancer and comprehensively summarizes the relationship between circRNAs and anticarcinogen resistance as well as underlying specific mechanisms in 14 types of cancer. First, an overview of current findings regarding the involvement of circRNAs in mediating resistance to anticarcinogens in cancer is presented; then, specific mechanisms by which circRNAs modulate anticarcinogen resistance in cancer are analyzed; finally, the potential prospects of circRNAs in the reversal of anticarcinogen resistance are discussed.

\section{Biogenesis of circRNAs}

CircRNAs are generally generated via back-splicing or exon skipping of pre-mRNAs, a process that differs from canonical splicing. A single genomic location can generate diverse types of circRNAs. CircRNAs are notable for their continuous closed loop structure, named a 'back-splicing' structure, which is primarily formed via the junction of a downstream $3^{\prime}$ splice site with an upstream $5^{\prime}$ splice site (head-to-tail splicing) [3]. Thus, these structures can resist exonucleolytic degradation by RNase R. In addition, exon skipping results in a restricted lariat structure that can promote cyclization. During the process of exonic circRNA (ecircRNA) biogenesis, direct back-splicing occurs more commonly than exon skipping [4]. Currently, circRNAs are considered to be divided into four categories on the basis of their composition: ecircRNAs; circular intronic RNAs (ciRNAs); exon-intron circRNAs (EIciRNAs); and tRNA intronic circular RNAs (tricRNAs), which are formed by tRNA introns. Over $80 \%$ of the identified circRNAs are ecircRNAs, and most are distributed in the cytoplasm [5,6]. However, ciRNAs as well as EIciRNAs are predominantly localized in the nucleus $[7,8]$, indicating that they may regulate gene transcription. Recently, researchers discovered another type of novel circular transcript called a read-through circRNA, which is formed by exons from the flanking regions of a gene via back-splicing [9].

To date, three hypothetical models for circRNA biogenesis mechanisms have been widely accepted: RBPmediated circularization; intron pairing-driven circularization; and lariat-driven circularization. The first model is called RBP-mediated circularization because RBPs have an important function in promoting circRNA biogenesis by regulating adjacent splice sites (Figure 1A). For instance, the splicing factors muscleblind (MBL) [10], quaking [11], adenosine deaminase acting on RNA 1 [12] and Asp-Glu-Ala-His (DEAH) box helicase 9 (DHX9) [13] are involved in circRNA formation. MBL can specifically bind with conserved MBL-binding sites in circMbl and its flanking introns [10]. Quaking can facilitate circRNA biogenesis by linking the flanking intron sequences and then connecting the adjacent introns [11]. Conversely, adenosine deaminase acting on RNA 1 may antagonize circRNA formation by destabilizing RNA pairing [12,14]. Similarly, because DHX9 contains both an RNA-binding domain and an RNA helicase domain, it can act as a nuclear RNA resolvase and may unwind RNA pairs flanking circularized exons to inhibit circRNA expression. Co-depletion of adenosine deaminase acting on RNA 1 and DHX9 can upregulate the expression of circRNAs [13], and heterogeneous nuclear ribonucleoprotein $\mathrm{L}$ and fused in sarcoma are also involved in regulating circRNA biogenesis $[15,16]$. In addition, the immune factors NF90 and NF110, which contain double-stranded RNA-binding domains, were observed to fuse with intronic RNA pairs to facilitate circRNA biogenesis [17].

Intron pairing-driven circularization is the second model of circRNA biogenesis (Figure 1B [18]). As pre-mRNA flanking introns contain inverted complementary sequences, alternative circularization can occur by complementary pairing of both sides of the introns, leading to the generation of various circRNAs, including ecircRNAs and EIciRNAs. Furthermore, longer introns can be found in the flanking sequences of circRNAs [6], and reverse complementary sequences in longer introns aid the formation of circRNAs [19].

During pre-mRNA transcription, an exon skipping event occurs that facilitates 'lariat-driven circularization' (Figure 1C). Subsequently, internal splicing facilitates the removal of the flanking intronic sequence, allowing the production of ecircRNAs [20]. However, under some circumstances, these sequences can be retained, and the resulting constructs are defined as EIciRNAs [8]. Moreover, this mechanism also facilitates ciRNA generation, a process that is facilitated by a ciRNA-specific consensus motif containing an 11-nt C-rich element near the branch point and a 7-nt GU-rich element near the 5' splice site (Figure 1C [7]). 


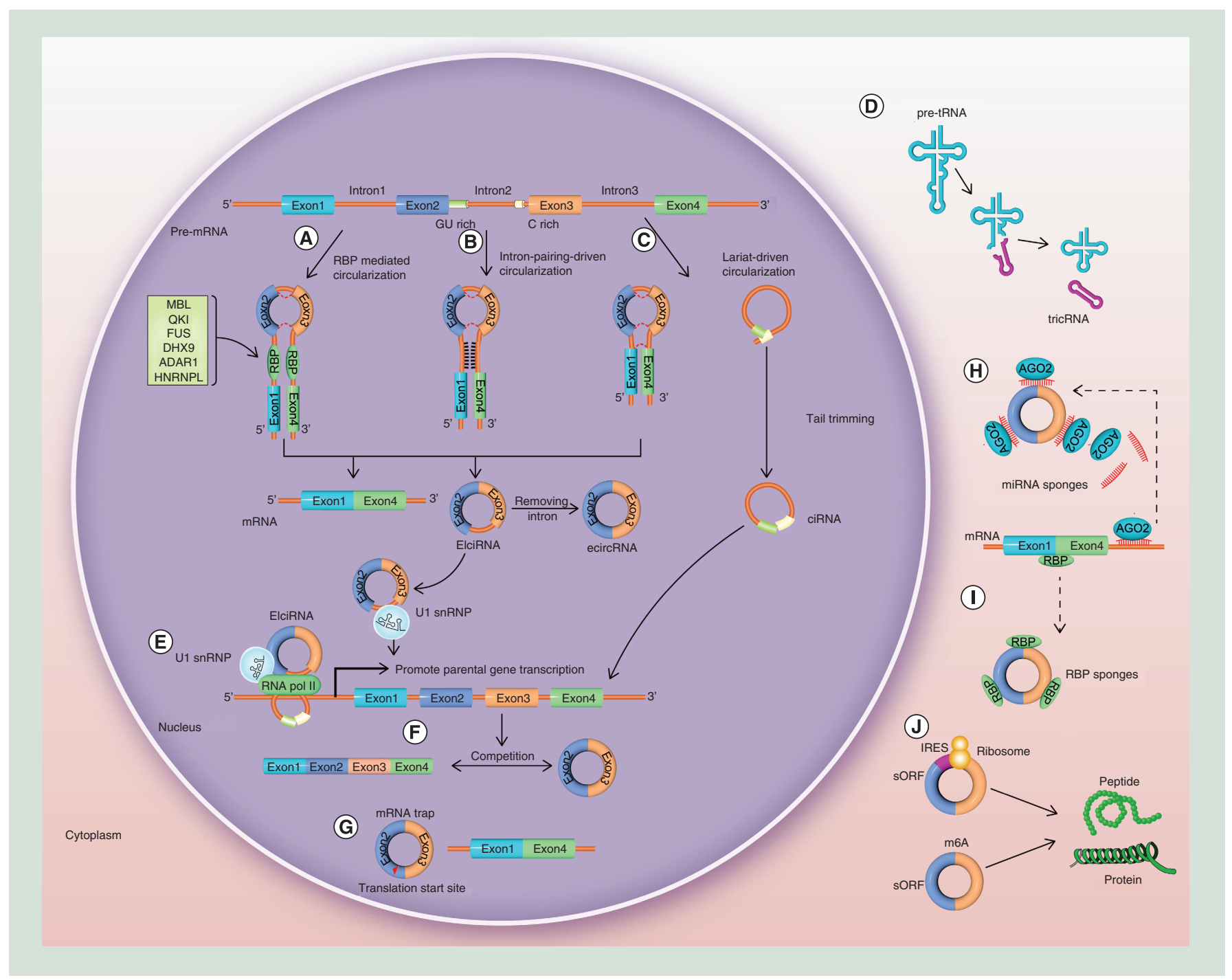

Figure 1. Circular RNA biogenesis mechanisms and the biological role of circular RNA. (A) RBP-mediated circularization. (B) Intron pairing-driven circularization. (C) Lariat-driven circularization. (D) TricRNAs are formed during the process of pre-tRNA splicing. (E) ElciRNAs can interact with U1 small nuclear ribonucleoproteins and then increase the transcription of their host genes by binding with RNA pol II; ciRNAs and the RNA pol II complex can directly interact and play a role in regulating parental gene transcription. (F) Circularization can compete with canonical splicing. (G) EcircRNAs can trap mRNAs. (H) CircRNAs can sponge miRNAs. (I) CircRNAs can act as protein sponges. (J) CircRNAs can be translated into peptides or proteins.

CiRNA: Circular intronic RNA; EcircRNA: Exonic circRNA; ElciRNA: Exon-intron circRNA; RBP: RNA-binding protein; RNA pol II: RNA polymerase II; TricRNA: tRNA intronic circular RNA.

TricRNAs are formed during the pre-tRNA splicing process. The bulge-helix-bulge motif of pre-tRNAs is recognized and spliced by the tRNA splicing endonuclease complex, and tricRNAs are then formed via combination of the released intron terminal ends (Figure 1D [21]).

\section{Biological functions of circRNAs}

Researchers worldwide are currently devoting increasing attention to circRNAs. Although no consensus has been reached on their biological functions, circRNAs have been confirmed to modulate parental gene expression, regulate alternative splicing and transcription as well as translation, act as miRNA sponges, act as RBP sponges or protein scaffolds, be translated into peptides/proteins (only a few circRNAs) and produce some pseudogenes. 


\section{CircRNAs can modulate parental gene expression}

CircRNAs can generate a vast repertoire of post-transcriptional regulators, as shown by Memczak et al. [5]. Some researchers have reported that some ciRNAs and EIciRNAs can modulate gene expression at both the transcriptional and post-transcriptional levels [22]. For instance, both circEIF3J and circPAIP2 are EIciRNAs and can interact with U1 small nuclear ribonucleoproteins, subsequently increasing host gene transcription by binding to RNA polymerase II $[7,8]$. Additionally, ciRNAs and the RNA polymerase II complex can directly interact to regulate parental gene transcription (Figure 1E). Interestingly, when EIciRNAs are knocked down, the transcription of their host genes decreases. These observations may explain why EIciRNAs and ciRNAs are found primarily in the nucleus.

Thus, specific circRNAs localized in the nucleus modulate parental gene expression as cis- or trans-acting factors at the transcriptional level. However, whether other circRNAs have these functions requires further exploration.

\section{CircRNAs can regulate alternative splicing \& transcription as well as translation}

Because circularization can compete with canonical splicing, ecircRNAs can play a role in alternative splicing. For example, the second exon of MBL was shown to form a circRNA by cyclization, which competes with linear splicing and impacts linear RNA formation to modulate the transcription of related genes (Figure 1F) [10]. So in cancer, aberrant transcription of oncogenes and tumor-suppressor genes can be promoted by the perturbated balance between circular and linear splicing.

Moreover, many single ecircRNAs in human fibroblasts contain a translation start site. EcircRNAs can be mRNA traps, inducing translation failure because of the lack of a translation start site. Therefore, ecircRNAs can play a vital role in regulating protein expression (Figure 1G) [4]. For example, through back-splicing, the mouse formin (Fmn) gene can create ecircRNAs that play a role as mRNA traps. As ecircRNAs cannot be generated from the Fmn site, the Fmn protein is abnormally expressed, and the associated phenotype is thereby changed [23].

Collectively, the circularization of forming circRNAs can regulate alternative splicing or the translation process of protein production. However, whether the occurrence of some disorders is related to this mechanism requires further clarification.

\section{CircRNAs can act as miRNA sponges}

Many circRNAs contain miRNA response elements that allow them to sponge miRNAs and act as ceRNAs in order to restrain miRNAs from negatively regulating their cognate target mRNAs [24]. By binding to the 3'UTR of mRNAs, miRNAs can suppress protein translation and facilitate mRNA degradation (Figure $1 \mathrm{H}$ ). Thus, circRNAs may indirectly regulate the translation of mRNAs.

Two research groups first described the roles of circRNAs as potent ceRNAs or miRNA sponges, roles that are most strongly exemplified by the function of CDR1as or the circRNA ciRS-7 [5,24]. However, shortly after this description was introduced, researchers revealed that some identified circRNAs contain fewer putative miRNAbinding sites than their co-linear mRNAs, and few circRNAs demonstrate the expected performance as miRNA sponges [25]. However, some circRNAs can also exhibit this sponge function despite lacking numerous miRNAbinding sites, indicating that the function of circRNAs as miRNA sponges is conserved across species. To date, multiple studies have demonstrated the sponge function of circRNAs; for example, circCSNK1G3 has been shown to promote prostate cancer (PCa) cell proliferation by interacting with miR-181b/d [26]. In addition, circHIPK3 suppresses miR-7 activity by sponging miR-7 [27]. Moreover, despite serving as miRNA sponges, circRNAs such as ciRS-7 can also stabilize miRNAs [28].

In summary, based on these results, circRNAs can commonly serve as miRNA sponges in the biological processes of cancer. Furthermore, circRNAs, miRNAs and mRNAs participate in a regulatory network that promotes the maintenance of cellular homeostasis.

\section{CircRNAs can act as protein sponges or protein scaffolds}

Specific circRNAs can act as protein sponges by providing binding sites for specific RBPs to sequester and inhibit the biological activity of proteins as competing elements (Figure 1I). For example, circPABPN1 and PABPN1 mRNA compete for binding to the HuR protein, and circPABPN1 binding with HuR can, accordingly, inhibit PABPN1 mRNA translation. Moreover, as demonstrated by their co-localization, circRNAs can interact with single or multiple RBPs [29]. CircRNAs are likely to act as protein scaffolds by harboring binding sites for the assembly of two or more proteins, such as enzymes and their substrates, which may subsequently form large protein 
complexes $[4,30]$. For example, by interacting with CDK2 and p21, circ-Foxo3 may generate a circ-Foxo3-p21CDK2 ternary complex, which then functions to suppress CDK2 [31]. CircYap (circBank ID: hsa_circYAP1_008) inhibits the initiation of Yap translation by binding to both eIF4G and PABP. Importantly, Yap activation can promote the proliferation, inhibit the apoptosis and facilitate the metastasis of cancer cells [32].

Although circRNAs can act as protein sponges, we do not know the extent to which low expression circRNAs may regulate the proteins they isolate or bind to.

\section{CircRNAs can be translated into proteins or peptides}

CircRNAs are considered ncRNAs due to the shortage of evidence for their translation; in addition, because they do not contain a 5' cap structure or a poly (A) tail, circRNAs cannot be translated into proteins through cap-dependent mechanisms. However, translation from pre-mRNAs, miRNAs, IncRNAs and other ncRNAs has been shown to occur from small open reading frames (sORFs), followed by the generation of functional peptides in vivo [33]. This potential activity demonstrates an obvious underestimation and disregard of the coding potential of these ncRNAs.

Using high-throughput technologies, an ORF was identified in circ-ZNF609, and this ORF can be translated via a splicing-dependent and cap-independent mechanism [34]. Additionally, circFBXW7 contains a putative ORF that can be translated into a novel 185-amino acid protein called FBXW7-185aa, which inhibits glioma proliferation and accelerates cell-cycle progression [35]. Zhang et al. [36] identified circ-SHPRH, which contains an internal ribosome entry site (IRES)-driven ORF for the translation of SHPRH-146aa, a functional protein in glioma. This research group also performed deep sequencing on ribosome nascent-chain complex-bound RNA, confirming that circPINTexon2 encodes an 87-amino acid peptide that suppresses tumors in human cells and verifying the independent biological role of this peptide in glioma [37]. Recently, Liang et al. [38] showed that circ $\beta$-catenin generates a new 370-amino acid $\beta$-catenin isoform that can regulate hepatocellular carcinoma (HCC) progression by activating the Wnt pathway. Presumably, ORFs harbored by circRNAs can be translated into peptides. Interestingly, both IRESs and N6-methyladenosine $\left(\mathrm{m}^{6} \mathrm{~A}\right)$ modification can drive the translation of circRNAs, further demonstrating that circRNAs have coding potential [39,40]. In 2017, Cell Research first published a study describing the $\mathrm{m}^{6} \mathrm{~A}$ modification of circRNA, noting that circRNAs contain an abundance of $\mathrm{m}^{6} \mathrm{~A}$ consensus motifs (accounting for $\sim 13 \%$ of the sequences) and that a single $\mathrm{m}^{6} \mathrm{~A}$ site, like an IRES, is enough to efficiently facilitate the initiation of circRNA translation. During this $\mathrm{m}^{6} \mathrm{~A}$-driven translation process, the $\mathrm{m}^{6} \mathrm{~A}$ reader YTHDF3 can recognize m6A modifications on circRNAs and then recruit translation initiation factors, including eIF4G2, to initiate protein translation. Moreover, the $\mathrm{m}^{6} \mathrm{~A}$ modification level can affect the translation efficiency of circRNAs [39]. Subsequently, researchers observed that $\mathrm{m}^{6} \mathrm{~A}$ modifications are widespread among circRNAs [41]. Recently, researchers established circRNADb, a circRNA database containing 32,914 human ecircRNAs that describes circRNAs in detail to assist users in predicting whether specific circRNAs can be translated; CircRNADb also includes IRES and ORF as well as genome sequence data (Figure 1J [42]).

The translation of circRNAs into peptides or proteins is a relatively novel and popular research topic, but due to the limitations of analysis and validation methods, only a few articles have reported on this phenomenon to date. Thus, this area of study requires further exploration.

\section{Pseudogenes derived from circRNAs}

Researchers developed a novel computational pipeline (CIRCpseudo) in 2016 to retrieve non co-linear back-splicing junction sequences, aiming to identify potential pseudogenes derived from circRNAs in the mouse reference genome. The pseudogenes containing the source of circRNAs in the mammalian genome were first identified. The circSATB1-derived pseudogene region can specifically bind to CCCTC-binding factor, suggesting that this circRNA-derived pseudogene can regulate gene expression. In addition, reverse transcription of circRNAs can drive pseudogene formation, as retrotransposed circRNAs can be inserted into the genome to alter the genome structure and regulate the potential for gene expression [43].

Under physiological and pathological conditions, pseudogenes can perform various functions. Future studies should focus on annotating pseudogenes derived from circRNAs in various species and profiling the expression modes of these pseudogenes in different transcriptomes, as well as explaining their functions.

\section{Degradation of circRNAs}

Compared with the mechanisms of circRNA biogenesis, the mechanisms by which cells ultimately degrade circRNAs remain largely unknown. Recently, the research group of Liu et al. [44] identified a secreted circRNA endonuclease, 
RNase L, which can globally degrade circRNAs. CircRNAs do not have free $5^{\prime}$ or $3^{\prime}$ ends, and endoribonucleolytic cleavage appears to be the only method of circRNA degradation. Based on the finding of Kim et al. [45], a subset of circRNAs containing $\mathrm{m}^{6} \mathrm{~A}$ are related to YTHDF2 in a manner dependent on HRSP12, which is preferentially downregulated via RNase P/MRP. $\mathrm{m}^{6} \mathrm{~A}$ can mediate the degradation of both mRNAs and circRNAs, a finding that appears to have increased researchers' recognition of this circRNA degradation mechanism [46].

\section{The mechanisms by which circRNAs play a role in cancer}

Hundreds of circRNAs have been shown to be abnormally expressed in different human cancers, but limited knowledge has been acquired about the mechanisms by which circRNAs play a role in cancer. It is increasingly understood that circRNAs play a significant role in cancer pathogenesis and progression.

Many studies have shown that circRNAs can affect cancer pathogenesis and progression by regulating target genes and multiple signaling pathways in a manner that acts as a miRNA sponge or another. For instance, by sponging miR-17 and miR-224, circ-ITCH is capable of regulation on p21 and phosphate and tensin homolog expression, which could induce cell cycle arrest and apoptosis [47]. Through sponging of miR-7, circHIPK3 inhibits miR-7 activity, which could cause an increase in the expression of FAK, IGF1R, EGFR and YYI oncogenes. As a kinase is crucial to cellular migration, FAK could trigger tumor invasion and metastasis through upregulated expression of VEGF, MMP2 and MMP9. Both IGF1R and EGFR are capable of activating PI3K/AKT and MEK/ERK signaling pathways, which could stimulate cancer progression and cause drug resistance. It has been reported that YY1 could be contributory to cancer growth through inhibition of P53 and activation of Wnt signaling pathways [48]. Besides, there is claim that Circ $\beta$-catenin could cause liver cancer cell to grow by activating the Wnt pathway [38]. CircFBXW7 can be translated into FBXW7-185aa, which could lead to a reduction in the half-life of c-Myc through antagonized stabilization of USP28-induced c-Myc. As a result, glioma proliferation is inhibited and cell-cycle progression is accelerated [35].

In summary, a variety of different pathways can be used by dysregulated circRNAs in cancer to achieve regulation, as a result of which proliferative signaling, epithelial-mesenchymal transition (EMT), angiogenesis and apoptosis or drug resistance can be affected. In this case, the progression of cancer can be impacted on directly.

\section{The potential role of circRNAs in mediating resistance to anticarcinogens}

Anticarcinogens play a crucial role in controlling tumor progression. However, many malignant tumors may eventually develop resistance to anticarcinogens. Clinically, drug resistance is a tremendous obstacle to cancer treatment and urgently needs a solution. Recently, many studies have revealed that ncRNAs are very important in various biological processes in tumors and have been implicated in resistance to various anticarcinogens $[1,49]$. Furthermore, many studies have demonstrated that circRNAs are associated with cancer formation, development, invasion and metastasis [50,51]. However, only a few studies have recently shown that circRNAs play a critical role in mediating resistance to anticarcinogens in cancer. The mechanism of circRNAs in anticarcinogen resistance has not been elucidated. In Table $1 \&$ Figure 2, we summarize currently known circRNAs associated with anticarcinogen resistance in 14 kinds of cancer.

\section{Non-small-cell lung cancer}

Lung cancer is an ordinary malignant tumor and is leading cause of cancer death worldwide. Chemotherapeutic resistance ultimately leads to poor therapy outcomes and recurrence of malignant lung cancer. Xu et al. [52] recently discovered upregulation of hsa_circ_0071799 in taxol-resistant non-small-cell lung cancer (NSCLC) cells, and also that hsa_circ_0071799 could play the role as a sponge of miR-141. Moreover, hsa_circ_0091931 was subject to downregulation and was associated with miR-34c-5p. Besides, P53 genes are possible to be targeted. The functions of circRNAs in Taxol chemoresistance in NSCLC were analyzed, and the results suggested that circRNAs may be involved in the regulation of Taxol resistance. Zhou et al. [53] investigated the differential expression of circRNAs in tyrosine kinase inhibitor (TKI)-resistant NSCLC cells and their corresponding parental cells. Analysis of clinical samples showed that hsa_circ_0004015 was upregulated in the tissues of NSCLC patients, and this upregulation predicted a poor prognosis. Overexpression of hsa_circ_0004015 in HCC827 cells can induce tolerance to gefitinib, and silencing hsa_circ_0004015 can increase the sensitivity of TKI-resistant NSCLC cells to gefitinib. Further studies showed that hsa_circ_0004015 can sponge miR-1183, which targets the PDPK1 gene, thus promoting gefitinib resistance in NSCLC cells. As revealed by Joseph et al. [54], the expression of circCCDC66 makes H23 cells more resistant to cisplatin (CDDP) through EMT. In addition to chemotherapeutic drugs, a study found that 


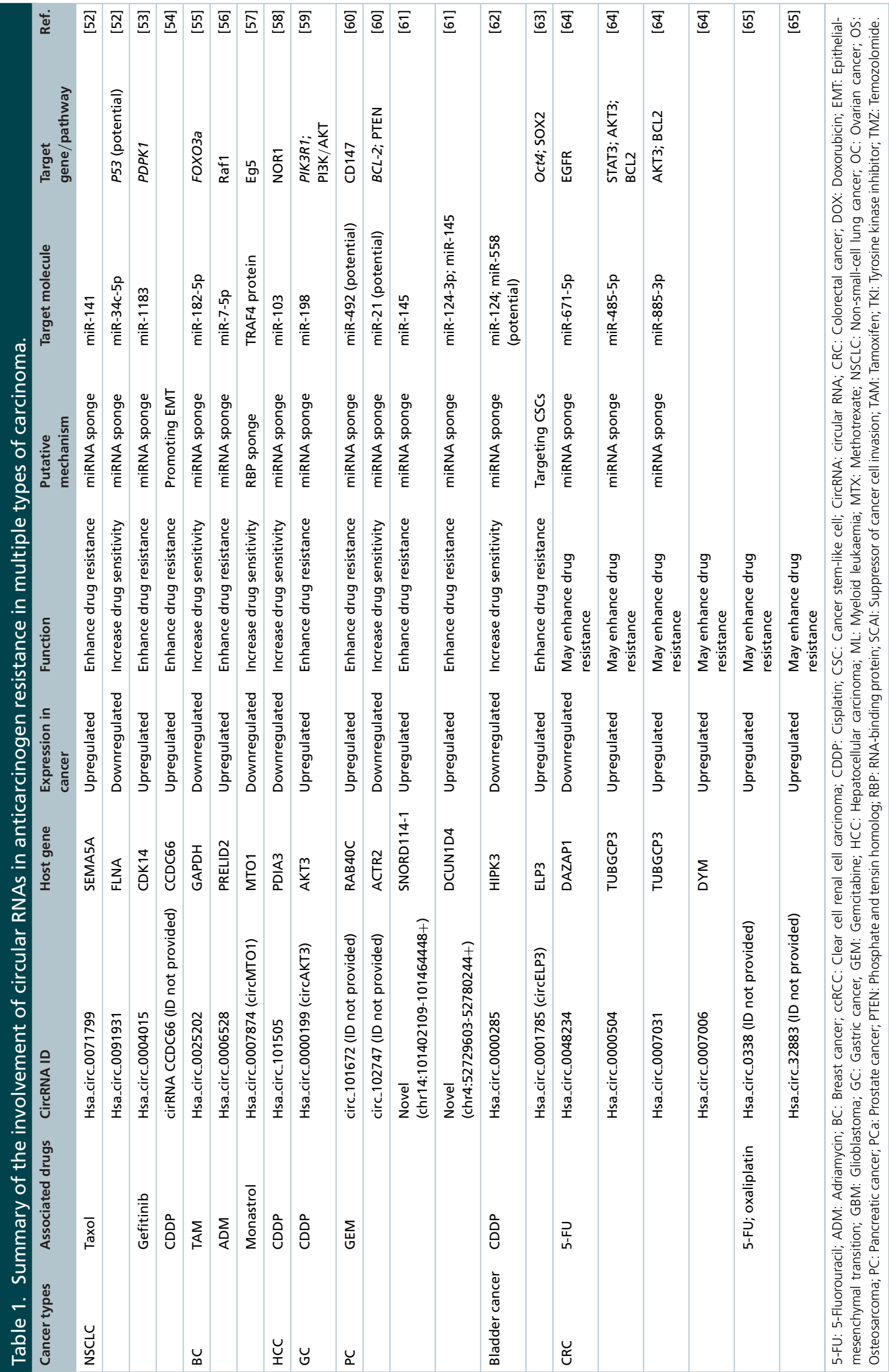




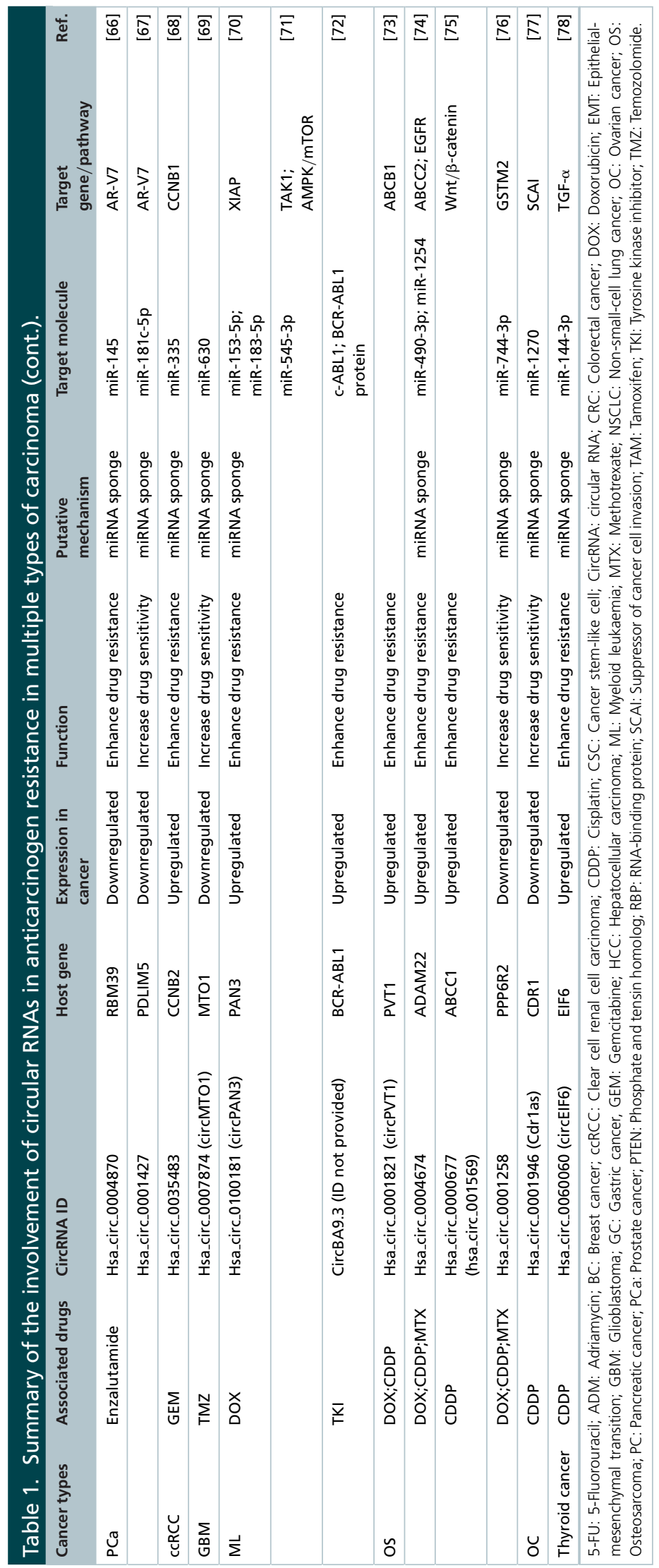




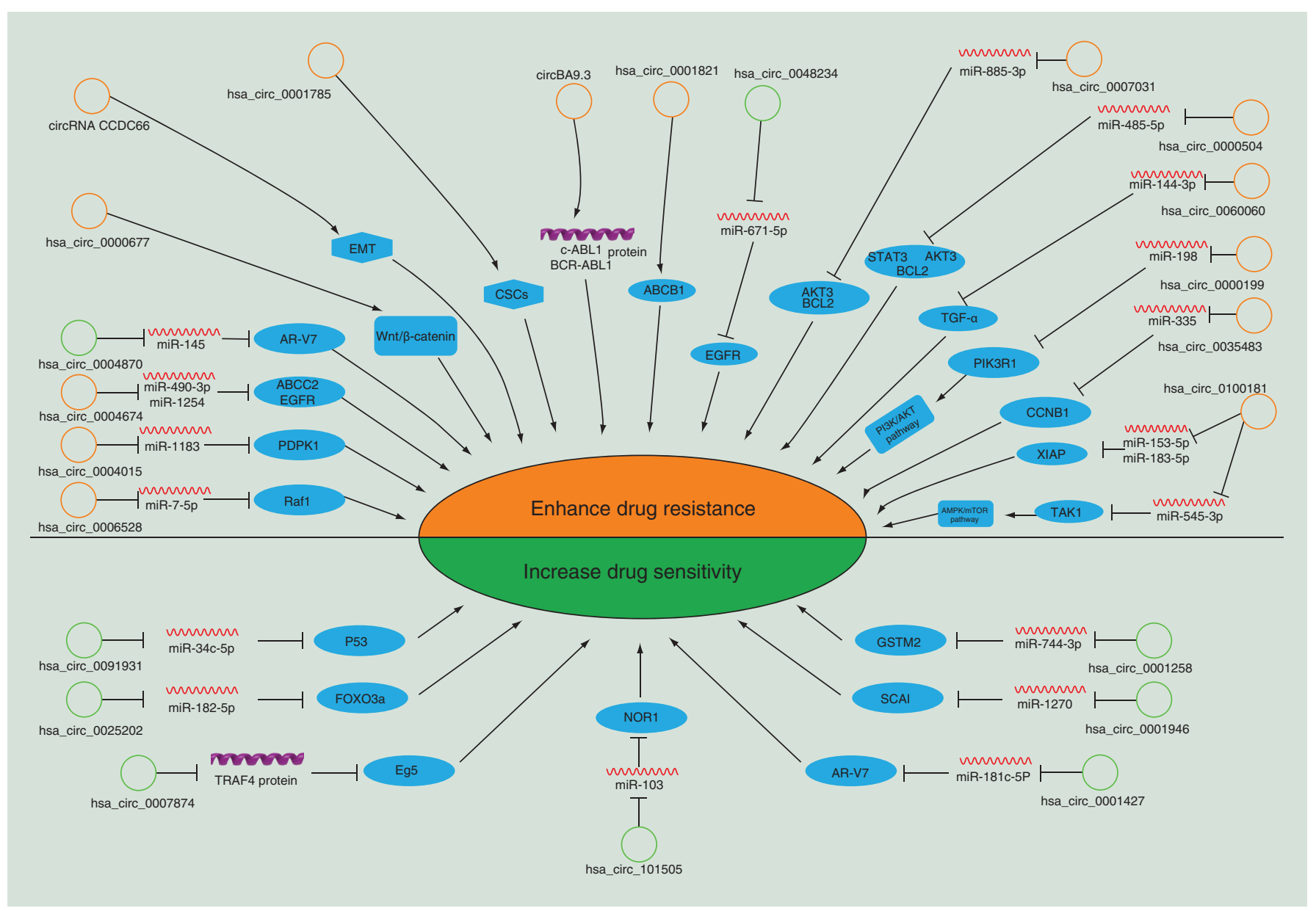

Figure 2. Regulatory network of circRNAs in mediating anticarcinogen resistance in multiple types of carcinoma.

dysregulated expression of circRNAs may have a critical function in mediating osimertinib resistance in NSCLC and could be candidate gene therapy targets [79].

\section{Breast cancer}

Breast cancer $(\mathrm{BC})$ is the second leading cause of death among women. Tamoxifen (TAM) is an important treatment option for BC. In one study, researchers established a TAM-resistant cell line and obtained the circRNA expression profile. Hsa_circ_0025202 inhibited cell proliferation, colony formation and migration, and promoted apoptosis and TAM sensitivity. Mechanistic investigations revealed that hsa_circ_0025202 can sponge miR-182-5p and that the expression and activity of the target gene $\mathrm{FOXO3a}$ were further modified [55]. According to another related study, differential expression of 18 circRNAs was found in adriamycin (ADM)-resistant MCF-7 human BC cells (MCF-7/ADM) compared with the corresponding parental MCF-7 cells. ADM-resistant human BC tissues and MCF-7/ADM cells exhibited significantly higher levels of hsa_circ_00006528 (circ_00006528) than the ADM-sensitive counterparts; however, circ_0006528 downregulation induced increased sensitivity to ADM in the ADM-resistant cell lines. Subsequent studies suggested that circ_0006528 may cause chemotherapeutic resistance in BC via the circ_0006528-miR-7-5p-Raf1 axis [56]. Studies by Liu et al. [57] revealed that the expression of the circRNA MTO1 (circMTO1) was downregulated in monastrol-resistant cells. Overexpression of the circMTO1 helped to decrease cell viability, facilitate cytotoxicity induced by monastrol and reverse monastrol resistance by preventing TRAF4 from binding to the Eg5 protein [57]. 
$\mathrm{HCC}$

HCC patients exhibit high rates of tumor metastasis and recurrence as well as drug and chemotherapy resistance; therefore, the prognosis of HCC is poor. A study was recently performed to assess the importance of circRNAs in mediating CDDP resistance in HCC and revealed a decrease in the level of circRNA_101505 in CDDP-resistant HCC tissues and cell lines. Based on the gain-of-function assay, circRNA_101505 overexpression inhibited the growth of cancer cells both in vitro and in vivo. As demonstrated by further research, by targeting miR-103, circRNA_101505 increased the sensitivity of HCC cells to CDDP, and miR-103 was predicted to target NOR1 [58].

\section{Gastric cancer}

Related studies have identified CDDP as well as fluorouracil (FU)-based chemotherapies as the first-line drugs for gastric cancer (GC) patients. As reported by Huang et al. [59], CDDP-resistant GC tissues and cells exhibit higher circAKT3 expression levels than their CDDP-sensitive counterparts. Upregulation of circAKT3 in GC patients who underwent CDDP treatment was clearly related to aggressive characteristics and was an independent risk factor for inferior disease-free survival. Additionally, hsa_circ_0000199 (circAKT3) promoted DNA damage repair and inhibited GC cell apoptosis. Consistent with the following mechanistic studies, circAKT3 could sponge miR-198 via the PI3K/AKT signaling pathway, thereby promoting the expression of PIK3R1.

\section{Pancreatic cancer}

Pancreatic cancer (PC) is one of the most malignant tumors. PDAC accounts for $85 \%$ of PC cases and carries a very poor prognosis. However, gemcitabine (GEM) resistance is a major obstacle to chemotherapy in patients with malignant PC.

With analysis conducted of the dysregulated circRNAs in the GEM-resistant PC cell line, a comparison was performed with its parental cell line. It is possible for circ_101672 and circ_102747 to bind some miRNAs associated with cancer chemoresistance [60]. Shao et al. [61] constructed the GEM-resistant PANC-1 (PANC-1-GR) cell line and compared the differential expression profiles of circRNAs between PANC-1 cells and PANC-1-GR cells through RNA sequencing. The results revealed a significant difference in the expression of 126 circRNAs between the two cell lines. Overexpression of two newly screened circRNAs, chr14:101402109-101464448+ and chr4:52729603-52780244+, increased GEM resistance and decreased the expression of miR-145, and silencing these circRNAs may assist in restoring GEM sensitivity - in other words, these circRNAs could be targets for combating GEM resistance.

\section{Bladder cancer}

Bladder cancer is a very challenging cancer due to its high rates of recurrence and progression, and its treatment is plagued by CDDP resistance. Hsa_circRNA_0000285 was found to be downregulated in tissues, cell lines and serum from bladder cancer patients; furthermore, its levels were lower in CDDP-resistant bladder cancer patients than in those who were CDDP-sensitive. These results indicate that hsa_circ_0000285 may be involved in bladder cancer chemosensitivity [62]. As discovered by Su et al. [63], hypoxia-elevation of hsa_circ_0001785 (circELP3) is effective in enhancing proliferation and CDDP resistance. However, this could cause the apoptosis of bladder cancer cells to be reduced. Reducing the level of circELP3 could inhibit the growth and CDDP resistance of bladder cancer cells while increasing apoptosis. Considering that cytoplasm is where circELP3 is concentrated, a prediction is made that circELP3 could make a difference by means of ceRNA activity. As revealed by further study, there was a noticeable upregulation of circELP3 expression, but the expression of stemness-related genes Oct4 and Sox 2 was reduced. Therefore, targeting cancer stem-like cells could enable circELP3 to increase CDDP resistance as well.

\section{Colorectal cancer}

Colorectal cancer (CRC) is a common type of cancer worldwide. However, resistance to 5-fluorouracil (5-FU) and oxaliplatin remains a major hurdle that needs to be conquered. Xiong et al. [64] provided the first report of the circRNA expression profiles in CRC cells resistant to 5-FU chemoradiation. Consistent with the microarray analysis results, 71 circRNAs exhibited differential expression in chemoradiation-resistant CRC cells; the expression of 47 circRNAs was upregulated and that of 24 was downregulated by more than twofold. The top three upregulated circRNAs were hsa_circ_0000504, hsa_circ_0007031 and hsa_circ_0007006. These upregulated circRNAs were hypothesized to be targets for reversing 5-FU resistance. Regarding the 24 downregulated circRNAs, 
hsa_circ_0048234 modulated the miR-671-5p-EGFR signaling pathway, thereby promoting chemoradiation resistance [64]. A newly reported study discovered 773 upregulated and 732 downregulated circRNAs between resistant HCT116 (HCT116-R) and parental HCT116 (HCT116-P) cells. The HCT116-R cell line exhibited resistance to 5-FU and oxaliplatin, commonly used chemotherapeutic drugs. Further assays revealed that hsa_circ_0338 and hsa_circ_32883 were upregulated in CRC cell lines and in 25 formalin-fixed paraffin-embedded tissues of primary CRC. These findings could provide insight into the functions of circRNAs in drug resistance in CRC [65].

\section{$\mathrm{PCa}$}

$\mathrm{PCa}$ is the second major cause of cancer-related death in males [80]. A study identified 111 circRNAs with differential expression in LNCaP clone 1 cells (highly resistant) and LNCaP clone 9 cells (moderately resistant) compared with LNCaP control cells (sensitive). In addition, enzalutamide-resistant cells exhibited downregulation of hsa_circ_0004870. Based on the results of further studies, hsa_circ_0004870 may be involved in the development of enzalutamide resistance in castration-resistant prostate cancer via the regulation of androgen receptor splicing variant 7 (AR-V7) [66]. Besides, it is speculated that hsa_circ_0001427 plays an inhibitory role to affect the enzalutamide sensitivity and cell invasion in castration-resistant prostate cancer cells. It is capable of making enzalutamide less resistant through regulation of the miR-181c-5p/AR-v7 signaling pathway [67].

\section{Clear cell renal cell carcinoma}

Clear cell renal cell carcinoma is a common renal cancer that exhibits drug resistance. Despite the great advantages of GEM in treating renal cancer, the current drug resistance issue remains an essential element affecting its treatment. Yan $e t a l$. [68] recently adopted the high-throughput sequencing approach to screen circRNAs abnormally expressed in renal cancer. According to their research, hsa_circ_0035483 acts as a miR-335 sponge to regulate autophagy, thereby promoting GEM resistance in human renal cancer cells and targeting the downstream CCNB1 mRNA. CircRNA expression can thus serve as a novel biomarker for clinical prognosis and a new target for combating cancer chemoresistance.

\section{Glioblastoma}

Temozolomide (TMZ) is the most commonly used chemotherapy drug in glioblastoma (GBM) patients. Despite initial good results, many patients eventually develop TMZ chemoresistance, which remains an acute clinical challenge. Rao et al. [69] found obvious downregulation of circMTO1 in TMZ-resistant GBM tissues and cells. As circMTO1 was overexpressed, GBM cells became markedly less resistant to TMZ in vitro and in vivo. In addition, when miR-630 was downregulated, TMZ resistance was attenuated. Mechanistically, circMTO1 can sponge miR-630, thereby reversing TMZ chemoresistance in GBM.

\section{Myeloid leukaemia}

Acute myeloid leukemia (AML) is a hematological malignancy. Primary or secondary drug resistance is inevitably acquired during the treatment of AML, leading to refractory disease and relapse. A related study adopted the highthroughput circRNA microarray approach for comparing the circRNA expression profiles in chemosensitive AML cells and doxorubicin (DOX)-resistant THP-1 AML cells. The study showed that hsa_circ_0100181 (circPAN3) can be an essential mediator of AML chemoresistance, possibly controlled by the circPAN3-miR-153-5p/miR183-5p-XIAP axis. As a key biomarker, circPAN3 can predict the clinical efficacy of chemotherapy in patients with AML and can also reverse drug resistance in AML as an underlying target [70]. This research group also found that circPAN3 can facilitate AML drug resistance by regulating autophagy and may sponge miR-545-3p and subsequently target $T A K 1$, ultimately activating the AMPK/mTOR signaling pathway [71].

In addition, TKIs are considered as an excellent drug treatment for the patients suffering chronic myelogenous leukemia. Nevertheless, there remain some patients showing no sensitivity to TKIs. In chronic myelogenous leukemia patients exhibiting TKI resistance, upregulation of circBA9.3 was observed. Through upregulation of protein expression for $\mathrm{c}-\mathrm{ABL} 1$ and BCR-ABL1, circBA9.3 could stimulate proliferation and suppress apoptosis of cancer cells, in addition to enhancing TKI resistance [72].

\section{Osteosarcoma}

Osteosarcoma (OS) is a common malignant bone tumor in children and adolescents. Patients who have poor sensitivity to chemotherapeutic drugs have relatively low 5-year survival rates. As reported by Zhu et al. [73], circPVT1 
upregulation modulated the ABCB1 expression level, thereby enhancing CDDP and DOX resistance. However, circPVT1 knockdown partially reversed the drug resistance effect. In addition, this group was the first to identify the comprehensive circRNA expression profile of three paired multidrug-resistant (DOX, CDDP and methotrexate) OS cell lines using next-generation sequencing technology. In chemoresistant OS patients, hsa_circ_0004674 was the most upregulated circRNA, and its upregulation was negatively correlated with prognosis; this circRNA is thus a potential therapeutic biomarker for chemoresistance in OS [74]. It was discovered by Zhang et al. [75] that OS showed a higher level of hsa_circ_0000677 (hsa_circ_001569) expression. By activation of Wnt/ $\beta$-catenin signaling pathway, the ability of cell proliferation was improved clearly by up-regulated hsa_circ_001569 and OS cell resistance to CDDP was also enhanced. Another research group found hsa_circ_0001258 can sponge miR-744-3p to upregulate the expression of GSTM2, thereby suppressing the resistance of OS cells to DOX [76].

\section{Ovarian cancer}

Ovarian cancer (OC) is the most common and the deadliest form of gynecologic malignancy. Apart from surgery, chemotherapy with CDDP is also a routine treatment for OS patients. However, CDDP chemoresistance restrict the therapeutic efficiency to a large extent. A microarray analysis was conducted by Zhao et al. [77] to explore how circRNAs are expressed in five paired CDDP-sensitive and CDDP-resistant tissues of OC. In CDDP-resistant patient tissues and cell lines, downregulation of hsa_circ_0001946 (Cdr1as) was discovered. Cell proliferation was repressed by the overexpression of Cdr1as and the CDDP-induced cell apoptosis in OC cells was promoted. Cdr1as suppress CDDP resistance in OC via functioning as miR-1270 sponge, and thereby upregulates suppressors of cancer cell invasion by partial abolition of its translational repression.

\section{Thyroid cancer}

As an aggressive type of thyroid cancer with a high-mortality rate, anaplastic thyroid cancer (ATC) shows a bad prognosis. Besides which, drug resistance of ATC restricts its therapeutic options. As revealed by Liu et al. [78], ATC tissues, papillary thyroid carcinoma and ATC cells could have an high expression of hsa_circ_0060060 (circEIF6). CircEIF6 is effective in making thyroid carcinoma more resistant to CDDP by acting as a sponge for miR-144-3p, which lead to an increased expression of TGF- $\alpha$, then promote autophagy and reduce cell apoptosis. On the contrary, circEIF6 knockdown could cause CDDP to be more sensitive.

\section{Specific mechanisms of circRNAs in anticarcinogen resistance}

According to current scientific research, we determined that miRNA sponging is the most common and important mechanism by which circRNAs play a role in mediating resistance to anticarcinogens. For example, circAKT3 could sponge miR-198 via the PI3K/AKT signaling pathway, thereby promote the expression of PIK3R1 [59]. CircPVT1, is capable of regulating multidrug resistance-related gene $A B C B 1$ (MDR1), which is effective in enhancing the chemoresistance. This is achieved by the P-gp protein which could facilitate the intracellular drugs to be pumped out [52]. It is possible that circPVT1 plays the role as miRNA sponges to increase the expression of $A B C B 1$. Despite this, further study is necessary to confirm. In addition, some circRNAs can also mediate anticarcinogen resistance by acting as RBP sponges, promoting EMT or targeting cancer stem-like cells. No matter which different mechanisms are involved, interestingly, it is found that such as circAKT3, circEIF6, circELP3 and circBA9.3, may facilitate anticarcinogen resistance by promoting proliferation, activating autophagy, inhibiting apoptosis and promoting DNA damage repair. The modulation of P53, FOXO3a, phosphate and tensin homolog and BCL2 gene, as well as several molecular pathways such as PI3K/AKT and Wnt/ $\beta$-Catenin play a role in these processes. When tumor cells continually proliferate to an extent, autophagy will increase, these cells escape apoptosis and develop resistance to anticarcinogens. In addition, the acquisition of stemness can promote tumor cells resistance to anticarcinogens. CircELP3 could facilitate CDDP resistance by mediating stemness-related genes Oct4 and Sox2. Apart from some stemness-related genes, some signaling pathways such as $\mathrm{Wnt} / \beta$-catenin may play a role in this process [63]. Nowadays, increasing studies reveal that EMT is closely related to anticarcinogen resistance. For example, circCCDC66 can increase the CDDP resistance by promoting EMT. Signaling pathways such as Wnt $/ \beta$-catenin and PI3K/AKT were reported associated with EMT [54].

\section{Conclusion}

The development of high-throughput sequencing technology has allowed multiple kinds of ncRNAs to be identified and validated to have a crucial function in cellular physiological and pathological processes. NcRNAs can regulate 
gene expression before, during and after transcription and are thus involved in regulating processes modulating tumor progression, such as cell proliferation, metastasis, migration, drug resistance and chemoresistance. CircRNA is a new kind of ncRNA, and the molecular mechanism of circRNA biogenesis and its biological functions relies on a complex regulatory network. To date, circRNAs have been reported to play important roles in the occurrence and progression of cancer. A variety of different pathways can be used by dysregulated circRNAs in cancer to achieve regulation, as a result of which proliferative signaling, EMT, angiogenesis and apoptosis or drug resistance can be affected. In this case, the progression of cancer can be impacted on directly. However, the key roles of circRNAs in mediating anticarcinogen resistance have seldom been systematically described. The important biological signaling pathway and the mechanisms and functions of circRNAs associated with anticarcinogen resistance need further exploration. Herein, we systematically described circRNAs that are involved in anticarcinogen resistance in 14 kinds of cancer. Current studies have shown that most circRNAs act as miRNA sponges in mediating resistance to anticarcinogens in cancer. These circRNAs can promote proliferation, activate autophagy, promote DNA damage repair and suppress tumor cell apoptosis, subsequently play a role in drug resistance. In addition, the acquisition of stemness and EMT are closely related to anticarcinogen resistance.

\section{Future perspective}

Understanding the new functions of circRNAs in anticarcinogen resistance or other aspects is only the tip of the iceberg. The miRNA sponge mechanism is just one of the many mechanisms of circRNAs, and exploring the other mechanisms and functions of circRNAs related to anticarcinogen resistance - for example, their functions in regulating parental genes, sponging RBPs and translating active proteins - is necessary. Being translated into proteins is a further example of another relatively novel and important function of circRNAs. Additional scientific studies are needed to explore other mechanisms by which circRNAs are involved in mediating anticarcinogen resistance. Abnormal circRNA expression in cancer may regulate the malignant progression of cancer via translation of active proteins. Whether these active proteins can be new targets for curbing anticarcinogen resistance is an important direction for future research. Besides, mediating stemness-related genes, promoting EMT and associated signaling pathways are also important mechanisms for circRNAs resistance to anticarcinogens, which also need to further investigate. Currently, only a few studies have addressed the role of circRNAs in anticarcinogen resistance, and most of these studies are single-center, small-sample studies that require future verification in multicenter, large-sample studies. In addition, most of the current scientific studies on circRNAs and anticarcinogen resistance are based at the cellular level, and evidence supporting the abnormal expression of circRNAs in the tumor tissues of clinically chemoresistant patient or in the tumor microenvironments is insufficient. Moreover, the current clinical evidence for treatment with circRNAs is still insufficient. More future clinical trials are needed if conditions permit. Thus, molecular targeted therapy of cancer and the use of therapeutic use of circRNAs or efficient small interfering RNAs targeting circRNAs are other current research direction. In conclusion, we believe that combining circRNAs with anticarcinogens is an effective strategy for the treatment of cancer.

\section{Author contributions}

XC Geng and YC Jia were the major contributors to the writing and revision of the manuscript. YH Zhang, L Shi and Q Li collected the related references and participated in discussions. All authors read and approved the final manuscript.

\section{Financial \& competing interest disclosure}

This work was financially supported by grants from the government-funded Provincial Clinical Medicine Talent Programs in 2017; Science and Technology Capacity Improvement Projects of Hebei University of Chinese Medicine in 2019 (no. KTZ2019019); Outstanding Student Scientific Research Ability Improvement Projects of Hebei University of Chinese Medicine in 2019 (no. YXZ2019002); Graduate Innovative Ability Training Projects of Hebei Province in 2020. The authors have no other relevant affiliations or financial involvement with any organization or entity with a financial interest in or financial conflict with the subject matter or materials discussed in the manuscript apart from those disclosed.

This paper was edited for proper English language, grammar, punctuation, spelling and overall style by one or more of the highly qualified native English-speaking editors at American Journal Experts. This certificate was issued on 28 September 2019 and may be verified on the American Journal Experts website using the verification code 1FB2-171E-2B93-202F-117P. 
Open access

This work is licensed under the Attribution-NonCommercial-NoDerivatives 4.0 Unported License. To view a copy of this license, visit http://creativecommons.org/licenses/by-nc-nd/4.0/

\section{Executive summary}

Background

- Noncoding RNAs are closely linked to most common diseases, especially human cancers, and are involved in mediating drug resistance in many kinds of cancer. However, only a few studies have reported that circRNAs have crucial functions in mediating resistance to anticarcinogens in cancer. The contribution of circRNAs to the development of anticarcinogen resistance in the context of tumors has not been fully described, and the mechanism of circRNAs in anticarcinogen resistance has not been elucidated.

Biogenesis of circRNAs

- CircRNAs are generally generated via back-splicing or exon skipping of precursor mRNAs. They are considered to be divided into four categories on the basis of their composition: exonic circRNAs; circular intronic RNAs; exon-intron circRNAs; and tRNA intronic circular RNAs, which are formed by tRNA introns. Now three hypothetical models for circRNA biogenesis mechanisms have been widely accepted: RNA-binding protein (RBP)-mediated circularization; intron pairing-driven circularization; and lariat-driven circularization.

Biological functions of circRNAs

- CircRNAs have been confirmed to modulate parental gene expression, regulate alternative splicing and transcription as well as translation, act as miRNA sponges, act as RBP sponges or protein scaffolds, be translated into peptides/proteins and produce some pseudogenes.

Degradation of circRNAs

- A research group identified a secreted circRNA endonuclease, RNase L, which can globally degrade circRNAs. In addition, $\mathrm{m}^{6} \mathrm{~A}$ can mediate the degradation of both mRNAs and circRNAs.

The mechanisms by which circRNAs play a role in cancer

- CircRNAs can affect cancer pathogenesis and progression by regulating target genes and multiple signaling pathways in a manner that acts as a miRNA sponge or another.

The potential role of circRNAs in mediating resistance to anticarcinogens

- CircRNAs associated with anticarcinogens resistance in 14 kinds of cancer were systematically described. Specific mechanisms of circRNAs in anticarcinogen resistance

- CircRNAs may act as related miRNA sponges or RBP sponges, and facilitate anticarcinogen resistance by promoting proliferation, activating autophagy, inhibiting apoptosis and promoting DNA damage repair. When tumor cells continually proliferate to an extent, autophagy will increase, these cells escape apoptosis and develop resistance to anticarcinogens. In addition, the acquisition of stemness can promote tumor cells resistance to anticarcinogens. CircRNAs can also increase anticarcinogen resistance by promoting epithelial-mesenchymal transition.

Conclusion

- The molecular mechanism of circRNA biogenesis and its biological functions relies on a complex regulatory network. In this review, we systematically describe circRNAs that are involved in anticarcinogen resistance in 14 kinds of cancer. Current studies have shown that most circRNAs act as miRNA sponges in mediating resistance to anticarcinogens in cancer. These circRNAs can promote proliferation, activate autophagy, promote DNA damage repair and suppress tumor cell apoptosis, subsequently play a role in drug resistance. In addition, the acquisition of stemness and epithelial-mesenchymal transition are closely related to anticarcinogen resistance.

Future perspective

- Understanding the new functions of circRNAs in anticarcinogen resistance or other aspects is only the tip of the iceberg. The miRNA sponge mechanism is just one of the many mechanisms of circRNAs, and exploring the other mechanisms and functions of circRNAs related to anticarcinogen resistance is necessary. The role of circRNAs in anticarcinogen resistance requires future verification in multicenter, large-sample studies. Evidence supporting the abnormal expression of circRNAs in the tumor tissues of clinically chemoresistant patient or in the tumor microenvironments is insufficient, and more future clinical trials are needed if conditions permit. In conclusion, we believe that combining circRNAs with anticarcinogens is an effective strategy for the treatment of cancer. 


\section{References}

Papers of special note have been highlighted as: • of interest; $\bullet \bullet$ of considerable interest

1. Xie C, Zhang LZ, Chen ZL et al. A novel hMTR4-PDIA3P1-miR-125/124-TRAF6 regulatory axis and its function in NF-KB signaling and chemoresistance. Hepatology doi:10.1002/hep.30931 (2019) (Epub ahead of print).

2. Qu L, Ding J, Chen C et al. Exosome-transmitted lncARSR promotes sunitinib resistance in renal cancer by acting as a competing endogenous RNA. Cancer Cell 29(5), 653-668 (2016).

- Describes that exosome mediate the transmition of drug resistance promoted by long noncoding RNA.

3. Zhang XO, Dong R, Zhang Y et al. Diverse alternative back-splicing and alternative splicing landscape of circular RNAs. Genome Res. 26(9), 1277-1287 (2016).

4. Jeck WR, Sharpless NE. Detecting and characterizing circular RNAs. Nat. Biotechnol. 32(5), 453-461 (2014).

5. Memczak S, Jens M, Elefsinioti A et al. Circular RNAs are a large class of animal RNAs with regulatory potency. Nature 495(7441), 333-338 (2013).

- The viewpoint that circular RNA (circRNA) could be amplified by divergent primers was first clarified.

6. Jeck WR, Sorrentino JA, Wang K et al. Circular RNAs are abundant, conserved, and associated with ALU repeats. RNA 19(2), 141-157 (2013).

7. Zhang Y, Zhang XO, Chen T et al. Circular intronic long noncoding RNAs. Mol. Cell 51(6), 792-806 (2013).

8. Li Z, Huang C, Bao C et al. Exon-intron circular RNAs regulate transcription in the nucleus. Nat. Struct. Mol. Biol. 22(3), 256-264 (2015).

9. Vo JN, Cieslik M, Zhang Y et al. The landscape of circular RNA in cancer. Cell 176(4), 869.e813-881.e813 (2019).

10. Ashwal-Fluss R, Meyer M, Pamudurti NR et al. circRNA biogenesis competes with pre-mRNA splicing. Mol. Cell 56(1), 55-66 (2014).

11. Conn SJ, Pillman KA, Toubia J et al. The RNA binding protein quaking regulates formation of circRNAs. Cell 160(6), 1125-1134 (2015).

12. Ivanov A, Memczak S, Wyler E et al. Analysis of intron sequences reveals hallmarks of circular RNA biogenesis in animals. Cell Rep. 10(2), 170-177 (2015).

13. Aktas T, Avsar Ilik I, Maticzka D et al. DHX9 suppresses RNA processing defects originating from the Alu invasion of the human genome. Nature 544(7648), 115-119 (2017).

14. Rybak-Wolf A, Stottmeister C, Glazar P et al. Circular RNAs in the mammalian brain are highly abundant, conserved, and dynamically expressed. Mol. Cell 58(5), 870-885 (2015).

15. Fei T, Chen Y, Xiao T et al. Genome-wide CRISPR screen identifies HNRNPL as a prostate cancer dependency regulating RNA splicing. Proc. Natl Acad. Sci. USA 114(26), E5207-E5215 (2017).

16. Errichelli L, Modigliani SD, Laneve P et al. FUS affects circular RNA expression in murine embryonic stem cell-derived motor neurons. Nat. Commun. 8, 14741 (2017).

17. Li X, Liu CX, Xue W et al. Coordinated circRNA biogenesis and function with NF90/NF110 in viral infection. Mol. Cell 67(2), 214.e217-227.e217 (2017).

18. Lee Y, Rio DC. Mechanisms and regulation of alternative pre-mRNA splicing. Annu. Rev. Biochem. 84, 291-323 (2015).

19. Zhang XO, Wang HB, Zhang Y, Lu X, Chen LL, Yang L. Complementary sequence-mediated exon circularization. Cell 159(1), 134-147 (2014).

20. Barrett SP, Wang PL, Salzman J. Circular RNA biogenesis can proceed through an exon-containing lariat precursor. Elife 4, e07540 (2015).

21. Noto JJ, Schmidt CA, Matera AG. Engineering and expressing circular RNAs via tRNA splicing. RNA Biol. 14(8), 978-984 (2017).

22. Salzman J, Gawad C, Wang PL, Lacayo N, Brown PO. Circular RNAs are the predominant transcript isoform from hundreds of human genes in diverse cell types. PLoS ONE 7(2), e30733 (2012).

23. Chao CW, Chan DC, Kuo A, Leder P. The mouse formin (Fmn) gene: abundant circular RNA transcripts and gene-targeted deletion analysis. Mol. Med. 4(9), 614-628 (1998).

24. Hansen TB, Jensen TI, Clausen BH et al. Natural RNA circles function as efficient microRNA sponges. Nature 495(7441), 384-388 (2013).

- $\quad$ First clarifies that circRNAs could function as miRNA sponges.

25. Guo JU, Agarwal V, Guo H, Bartel DP. Expanded identification and characterization of mammalian circular RNAs. Genome Biol. 15(7), 409 (2014).

26. Chen S, Huang V, Xu X et al. Widespread and functional RNA circularization in localized prostate cancer. Cell 176(4), 831.e822-843.e822 (2019).

27. Zeng K, Chen X, Xu M et al. CircHIPK3 promotes colorectal cancer growth and metastasis by sponging miR-7. Cell Death Discov. 9(4), 417 (2018). 
28. Piwecka M, Glazar P, Hernandez-Miranda LR et al. Loss of a mammalian circular RNA locus causes miRNA deregulation and affects brain function. Science 357(6357), eaam8526 (2017).

29. Abdelmohsen K, Panda AC, Munk R et al. Identification of HuR target circular RNAs uncovers suppression of PABPN1 translation by CircPABPN1. RNA Biol. 14(3), 361-369 (2017).

30. Hansen TB, Veno MT, Damgaard CK, Kjems J. Comparison of circular RNA prediction tools. Nucleic Acids Res. 44 (6), e58 (2016).

31. Du WW, Yang W, Liu E, Yang Z, Dhaliwal P, Yang BB. Foxo3 circular RNA retards cell cycle progression via forming ternary complexes with p21 and CDK2. Nucleic Acids Res. 44(6), 2846-2858 (2016).

32. Wu N, Yuan Z, Du KY et al. Translation of yes-associated protein (YAP) was antagonized by its circular RNA via suppressing the assembly of the translation initiation machinery. Cell Death Differ. doi:10.1038/s41418-019-0337-2 (2019) (Epub ahead of print).

33. Lauressergues D, Couzigou JM, Clemente HS et al. Primary transcripts of microRNAs encode regulatory peptides. Nature 520(7545), 90-93 (2015)

34. Legnini I, Di Timoteo G, Rossi F et al. Circ-ZNF609 is a circular RNA that can be translated and functions in myogenesis. Mol. Cell. 66(1), 22-37.e29 (2017).

35. Yang Y, Gao X, Zhang M et al. Novel role of FBXW7 circular RNA in repressing glioma tumorigenesis. J. Natl Cancer Inst. 110(3), (2018).

36. Zhang M, Huang N, Yang X et al. A novel protein encoded by the circular form of the SHPRH gene suppresses glioma tumorigenesis. Oncogene 37(13), 1805-1814 (2018).

37. Zhang M, Zhao K, Xu X et al. A peptide encoded by circular form of LINC-PINT suppresses oncogenic transcriptional elongation in glioblastoma. Nat. Commun. 9(1), 4475 (2018).

38. Liang WC, Wong CW, Liang PP et al. Translation of the circular RNA circbeta-catenin promotes liver cancer cell growth through activation of the Wnt pathway. Genome Biol. 20(1), 84 (2019).

39. Yang Y, Fan X, Mao M et al. Extensive translation of circular RNAs driven by N(6)-methyladenosine. Cell Res. 27(5), 626-641 (2017).

40. Pamudurti NR, Bartok O, Jens M et al. Translation of CircRNAs. Mol. Cell. 66(1), 9.e27-21.e27 (2017).

41. Zhou C, Molinie B, Daneshvar K et al. Genome-wide maps of m6A circRNAs identify widespread and cell-type-specific methylation patterns that are distinct from mRNAs. Cell Rep. 20(9), 2262-2276 (2017).

42. Chen X, Han P, Zhou T, Guo X, Song X, Li Y. circRNADb: a comprehensive database for human circular RNAs with protein-coding annotations. Sci. Rep. 6, 34985 (2016).

43. Dong R, Zhang XO, Zhang Y, Ma XK, Chen LL, Yang L. CircRNA-derived pseudogenes. Cell Res. 26(6), 747-750 (2016).

44. Liu CX, Li X, Nan F et al. Structure and degradation of circular RNAs regulate PKR activation in innate immunity. Cell 177(4), 865.e821-880.e821 (2019).

45. Kim SM, Yang Y, Oh SJ, Hong Y, Seo M, Jang M. Cancer-derived exosomes as a delivery platform of CRISPR/Cas9 confer cancer cell tropism-dependent targeting. J. Control. Release 266, 8-16 (2017).

46. Park OH, Ha H, Lee Y et al. Endoribonucleolytic cleavage of m(6)A-containing RNAs by RNase P/MRP complex. Mol. Cell 74(3), 494.e498-507.e498 (2019).

47. Yang C, Yuan W, Yang X et al. Circular RNA circ-ITCH inhibits bladder cancer progression by sponging miR-17/miR-224 and regulating p21, PTEN expression. Mol. Cancer 17(1), 19 (2018).

48. Zeng K, Chen X, Xu M et al. CircHIPK3 promotes colorectal cancer growth and metastasis by sponging miR-7. Cell Death Dis. 9(4), 417 (2018).

49. Liu T, Zhang X, Du L et al. Exosome-transmitted miR-128-3p increase chemosensitivity of oxaliplatin-resistant colorectal cancer. Mol. Cancer 18(1), 43 (2019).

50. Zhang PF, Wei CY, Huang XY et al. Circular RNA circTRIM33-12 acts as the sponge of MicroRNA-191 to suppress hepatocellular carcinoma progression. Mol. Cancer 18(1), 105 (2019).

51. Dong W, Bi J, Liu H et al. Circular RNA ACVR2A suppresses bladder cancer cells proliferation and metastasis through miR-626/EYA4 axis. Mol. Cancer 18(1), 95 (2019).

52. Xu N, Chen S, Liu Y et al. Profiles and bioinformatics analysis of differentially expressed circrnas in taxol-resistant non-small cell lung cancer cells. Cell Physiol. Biochem. 48(5), 2046-2060 (2018).

53. Zhou Y, Zheng X, Xu B et al. Circular RNA hsa_circ_0004015 regulates the proliferation, invasion, and TKI drug resistance of non-small cell lung cancer by miR-1183/PDPK1 signaling pathway. Biochem. Biophys. Res. Commun. 508(2), 527-535 (2019).

54. Joseph NA, Chiou SH, Lung Z et al. The role of HGF-MET pathway and CCDC66 cirRNA expression in EGFR resistance and epithelial-to-mesenchymal transition of lung adenocarcinoma cells. J. Hematol. Oncol. 11(1), 74 (2018).

55. Sang $\mathrm{Y}$, Chen B, Song X et al. circRNA_0025202 regulates tamoxifen sensitivity and tumor progression via regulating the miR-182-5p/FOXO3a axis in breast cancer. Mol. Ther. 27(9), 1638-1652 (2019).

-. One of the important studies on circRNA and drug resistance in breast cancer. 
56. Gao D, Zhang X, Liu B et al. Screening circular RNA related to chemotherapeutic resistance in breast cancer. Epigenomics 9(9), 1175-1188 (2017).

57. Liu Y, Dong Y, Zhao L, Su L, Luo J. Circular RNAMTO1 suppresses breast cancer cell viability and reverses monastrol resistance through regulating the TRAF4/Eg5 axis. Int. J. Oncol. 53(4), 1752-1762 (2018).

58. Luo Y, Fu Y, Huang R et al. CircRNA_101505 sensitizes hepatocellular carcinoma cells to cisplatin by sponging miR-103 and promotes oxidored-nitro domain-containing protein 1 expression. Cell Death Discov. 5, 121 (2019).

59. Huang X, Li Z, Zhang Q et al. Circular RNA AKT3 upregulates PIK3R1 to enhance cisplatin resistance in gastric cancer via miR-198 suppression. Mol. Cancer 18(1), 71 (2019).

-. One of the important papers on circRNA and chemoresistance in gastric cancer.

60. Xu C, Yu Y, Ding F. Microarray analysis of circular RNA expression profiles associated with gemcitabine resistance in pancreatic cancer cells. Oncol. Rep. 40(1), 395-404 (2018).

61. Shao F, Huang M, Meng F, Huang Q. Circular RNA signature predicts gemcitabine resistance of pancreatic ductal adenocarcinoma. Front. Pharmacol. 9, 584 (2018).

62. Chi BJ, Zhao DM, Liu L et al. Downregulation of hsa_circ_0000285 serves as a prognostic biomarker for bladder cancer and is involved in cisplatin resistance. Neoplasma 66(2), 197-202 (2019).

63. Su Y, Yang W, Jiang N et al. Hypoxia-elevated circELP3 contributes to bladder cancer progression and cisplatin resistance. Int. J. Biol. Sci. 15(2), 441-452 (2019).

64. Xiong W, Ai YQ, Li YF et al. Microarray analysis of circular RNA expression profile associated with 5-fluorouracil-based chemoradiation resistance in colorectal cancer cells. Biomed. Res. Int. 2017, 8421614 (2017).

65. Abu N, Hon KW, Jeyaraman $\mathrm{S}$ et al. Identification of differentially expressed circular RNAs in chemoresistant colorectal cancer. Epigenomics 11(8), 875-884 (2019).

66. Greene J, Baird AM, Casey O et al. Circular RNAs are differentially expressed in prostate cancer and are potentially associated with resistance to enzalutamide. Sci. Rep. 9(1), 10739 (2019).

67. Wu G, Sun Y, Xiang Z et al. Preclinical study using circular RNA 17 and micro RNA 181c-5p to suppress the enzalutamide-resistant prostate cancer progression. Cell Death Dis. 10(2), 37 (2019).

68. Yan L, Liu G, Cao H, Zhang H, Shao F. Hsa_circ_0035483 sponges hsa-miR-335 to promote the gemcitabine-resistance of human renal cancer cells by autophagy regulation. Biochem. Biophys. Res. Commun. 519(1), 172-178 (2019).

69. Rao J, Cheng X, Zhu H, Wang L, Liu L. Circular RNA-0007874 (circMTO1) reverses chemoresistance to temozolomide by acting as a sponge of microRNA-630 in glioblastoma. Cell Biol. Int. doi:10.1002/cbin.11080 (2018) (Epub ahead of print).

70. Shang J, Chen WM, Wang ZH, Wei TN, Chen ZZ, Wu WB. CircPAN3 mediates drug resistance in acute myeloid leukemia through the miR-153-5p/miR-183-5p-XIAP axis. Exp. Hematol. 70, 42.e43-54.e43 (2019).

71. Shang J, Chen WM, Liu S et al. CircPAN3 contributes to drug resistance in acute myeloid leukemia through regulation of autophagy. Leuk. Res. 85, 106198 (2019).

72. Pan Y, Lou J, Wang $\mathrm{H}$ et al. CircBA9.3 supports the survival of leukaemic cells by up-regulating c-ABL1 or BCR-ABL1 protein levels. Blood Cells Mol. Dis. 73, 38-44 (2018).

73. Kun-Peng Z, Xiao-Long M, Chun-Lin Z. Overexpressed circPVT1, a potential new circular RNA biomarker, contributes to doxorubicin and cisplatin resistance of osteosarcoma cells by regulating ABCB1. Int. J. Biol. Sci. 14(3), 321-330 (2018).

74. Kun-Peng Z, Xiao-Long M, Lei Z, Chun-Lin Z, Jian-Ping H, Tai-Cheng Z. Screening circular RNA related to chemotherapeutic resistance in osteosarcoma by RNA sequencing. Epigenomics 10(10), 1327-1346 (2018).

- First identifies the circRNA expression profile of three-paired multidrug-resistant osteosarcoma cell lines.

75. Zhang H, Yan J, Lang X, Zhuang Y. Expression of circ_001569 is upregulated in osteosarcoma and promotes cell proliferation and cisplatin resistance by activating the Wnt/beta-catenin signaling pathway. Oncol. Lett. 16(5), 5856-5862 (2018).

76. Zhu KP, Zhang CL, Ma XL, Hu JP, Cai T, Zhang L. Analyzing the interactions of mRNAs and ncRNAs to predict competing endogenous RNA networks in osteosarcoma chemo-resistance. Mol. Ther. 27(3), 518-530 (2019).

77. Zhao Z, Ji M, Wang Q, He N, Li Y. Circular RNA Cdrlas upregulates SCAI to suppress cisplatin resistance in ovarian cancer via miR-1270 suppression. Mol. Ther. Nucleic Acids 18, 24-33 (2019).

78. Liu F, Zhang J, Qin L et al. Circular RNA EIF6 (Hsa_circ_0060060) sponges miR-144-3p to promote the cisplatin-resistance of human thyroid carcinoma cells by autophagy regulation. Aging 10(12), 3806-3820 (2018).

79. Chen T, Luo J, Gu Y, Huang J, Luo Q, Yang Y. Comprehensive analysis of circular RNA profiling in AZD9291-resistant non-small cell lung cancer cell lines. Thorac. Cancer 10(4), 930-941 (2019).

80. Fenton JJ, Weyrich MS, Durbin S, Liu Y, Bang H, Melnikow J. Prostate-specific antigen-based screening for prostate cancer: evidence report and systematic review for the US preventive services task force. JAMA 319(18), 1914-1931 (2018). 
